\title{
XVII.
}

\section{Einige Bemerkungen über den sogenannten Harnsäureinfarct in den Nieren neugeborner Kinder.}

\author{
Von Dr. C. Hecker, Privatdocent in Berlin.
}

\begin{abstract}
Mit der Frage über die Abscheidung von harnsauren Salzen in den Nieren neugeborner Kinder haben sich, seitdem die Thatsache durch Cless constatirt worden, so bedeutende Forscher beschäftigt, und erst ganz vor Kurzem ist der Gegenstand von Hodann*) so erschöpfend und mit so sorgfältiger Benutzung der einschlägigen Literatur bearbeitet worden, dass es überflüssig erscheinen könnte, denselben schon jetzt wieder aufzunehmen, wenn sich nicht in den gelieferten Arbeiten mehrfach die Forderung ausgesprochen fände, dass eine sich darbietende Gelegenheit zu wiederholter Prüfung der bisher gewonnenen Resultate nicht verabsäumt werden solle. Auf diese Veranlassung hin habe ich längere Zeit mein Augenmerk auf das uropoetische System der Neugebornen gerichtet, und mich vielfach bemübt, Anhaltspunkte zur Lösung der immer noch in verschiedenem Sinne beantworteten Frage zu gewinnen, ob die Ausscheidung von harnsaurem Natron in die Nierenkanäle ein innerhalb einer gewissen Zeit nach der Geburt nothwendig und immer eintretender Vorgang ist, oder ob zum Zustandekommen derselben abnorme Bedingungen erfordert werden. Hierbei habe ich nun sehr bald die Ueberzeugung gewonnen, dass der Weg, den man bisher vielfach verfolgt hat, aus dem Vorhandensein oder der Abwesenheit des Niereninfarctes bei Sectionen neugeborner Kinder auf statistischem Wege diesen Punkt zu entscheiden, nicht zum Ziele führt, denn so lange nicht Obductionen von Kindern vorliegen, welcbe, vorher unzweifelhaft gesund, emes plötzliehen gewaltsamen Todes gestorben sind, und bei denen man

*) J. Hodann, Der Harnsäureinfarct in den Nieren neugeborner Kinder in seiner plysiologisclen, pathologischen und forensischen Bedeutung. Breslau 18 j̃j.

Archiv C. pathol. Anat. Bd. XI. Hf. 3.


harnsaure Salze in den Nieren gefunden hat, so kann von denjenigen, welche die physiologische Natur des Infaretes behaupten, immer der Einwand nicht entkräftet werden, dass die gewöhnlichen Sectionen an Kindern gemacht worden, wo Gesundheit und Tod nicht zusammenfielen, sondern durch eine Krankheit, also durch eine kürzere oder längere Existenz des Organismus unter veränderten Bedingungen von einander getrennt waren. Auch ist in den früheren Arbeiten zur Genüge hervorgehoben worden, dass sich aus der Zusammenstellung der verschiedenen Krankheiten, welche die Obduction als mit Harnsäureinfarct combinirt, oder ohne denselben vorfindig nachgewiesen hat, keine beachtenswerthe Ausbeute für die Aetiologie des letzteren ergiebt; namentlich sind die Beobachtungen über das Nichtrorhandensein desselben höchst unsicher, weil schon vor dem Tode die Nierenkanälchen zum Theil oder ganz von ihrem Inhalt befreit sein können, und man bei der Obduction höchstens noch in der Blase, bei männlichen Kindern an der Spitze des Penis, oder auch dort nicht mehr die Sedimente findet; es kann also sehr wohl die Krankheit mit Abscheidung von Uraten in die Nieren einhergegangen sein, ohne dass die Obduction daruber den Nachweis zu liefern im Stande ist. Wenn ich dennoch Sectionsresultate hier folgen lasse, so geschieht dies eigentlich nur, um zu zeigen, dass überhaupt eine solche Zahl kindlicher Leichen durch meine Hände gegangen ist, welche eine wiederholte Prufung des Gegenstandes gestattet hat.

Von October 1852 bis September 1856, also in einem Zeitraum von 4 Jahren, sind in der Königl. Entbindungs-Anstalt 211 neugeborne kinder theils von meinen Collegen, theils von mir obducirt, und über den Befund mehr oder weniger genaue Notizen aufgenommen worden. Unter diesen waren freilich 115 todtgeborne, von denen nur eines Harnsäureinfarct aufwies (der Hoogewe g'sche Fall); es bleiben aber immer noch $97 \mathrm{zu}$ verschiedenen Zeiten nach der Geburt verstorbene übrig, deren Obduction in Bezug auf den Befund in den Nieren folgende Resultate ergeben hat: bei 39 wurden in geringerem oder stärkerem Grade harnsaure Salze in den Nieren gefunden, bei 57 fehlten sie und zwar ordnen sich diese Fulle nach der Zeit des extrauterinen Lebens folgendermaassen: 
Harnsắureinfarct vorkanden

10 Stund. nach der Geburt 1

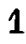

1

38

48

$60-$

3 Tage

4

5

6

7

8

9

10 -

11 -

13 -

$19-$

25 -

29 -

44 -

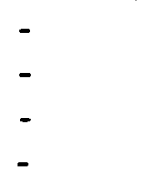

- 2

- 5

$-\quad 5$

- 5

$-\quad 1$

- 1

- 1

- 3

- 3

- 1

- 3

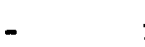

- 1

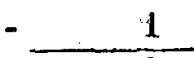

Suintna 39.
Fehlend

12 Stund. nach der Geburt 9

124 -

136 -

2 Tage

3 -

4.

5 -

7

8

9

11 .

13

14

18

19

21

22

24

27

31.

35

2 Monate

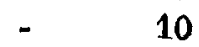

- 4

- 2

- 2

- 1

- 2

- 2

- 1

- 2

- 2

- 3

- 4

- 2

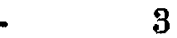

- 1

- 2

- 1

- 1

- 1

- 1

SuImusa $\frac{1}{57 .-}$

Wenn man diese Angaben auf die von Hodann in seiner angeführten Schrift Seite 14 zusammengestellte Tabelle reducirt, so erhält man folgende Zahlen:

Bald nach der Geburt gest. 21 davon 2 mit Infarct 19 ohne Infarct. Bis zum 2ten Tage gest. $9-3$ - 6 Vom $2-8$ Tage gest. $28-20-8$ $-8-14--19-8-11$. $-14-21,-9-3-6-$

$-21-30--6-2-4-$

Summe der Sectionen $96-30-60-39-37-$ 
Es wird hiernach leicht sein zu sehen, ob die Procentangaben, welche der genannte Schriftsteller aus seiner Tabelle entnimmt, durch unsere Zahlen modificirt werden oder nicht.

1) Nach ihm fand sich bei 31 bald nach der Geburt gestorbenen Kindern der Infarct $1 \mathrm{mal}(4 \mathrm{pCt}$.); fugen wir unsre 21 Fälle hinzu, unter denen 2 mit Infaret, so kommen auf 52 Fälle 3 mit Infarct ( 6 pCt.).

2) Bei 51 im Verlauf des 1sten Tages gestorbenen 9 mit Infarct $(18 \mathrm{pCt}$.), mit Hinzurechnung von 9 , wovon 3 positive, hommen auf 60 Fälle 12 mit Infarct $(20 \mathrm{pCt}$.).

3) Von 157 zwischen dem 2ten und 14 ten Tage gestorbenen hatten 81 Infarct ( $52 \mathrm{pCt}$.), dazu 47 , wovon 28 mit Infarct, giebt 204 mit 109 Infarct (53 pCt.).

- 4) $\mathrm{Zu} 74$ Kindern, welche vom 14 ten bis 60 sten Tage starben und von denen 27 Infarct hatten ( $37 \mathrm{pCt}$.), kommen 19 hinzu, davon 6 mit Infarct. Man bekommt also von 93 Kindern 33 mit Infarct $(36,5 \mathrm{pCt}$.).

Es ergiebt sich hieraus, dass im Wesentlichen die Resultate der Hodann'schen Tabelle durch unsre Zahlen nicht alterirt werden, namentlich wird die Thatsache, dass mehr als die Hälfte der Kinder, welche vom 2ten bis 14ten Tage nach der Geburt zur Section kommen, den Niereninfarct darbieten, aufs Neue bestätigt. Der spăteste Termin, bis zu welchem derselbe gesehen worden ist, 44. Tage nach der Geburt, liegt innerhalb der von Hodann aufgefundenen Grenze, da er ihn in einem Falle noch am 60sten Tage beobachtet hat; dagegen ist der früheste Termin (Tod des Kindes 10 Stunden nach der Geburt) in seiner präcisen Angabe ohne Analogie, da das von Martin obducirte Kind 18 Stunden nach der Geburt gestorben war, und die Angaben Schlossberger's sich nur im Allgemeinen auf den ersten Tag des extrauterinen Lebens beziehen, innerhalb welches er 6 mal den Infarct fand. Der betreffende Fall ist von anderweitigem Interesse, und wird desshalb weiter unten mitgetheilt werden.

In der Ueberzeugung, die oben schon ausgesprochen ist, dass aus der Zusammenstellung der krankhaften Veränderungen, welche bei den Sectionen in den verschiedenen Organen constatirt werden 
konnten, in Bezug auf die vorliegende Frage kein Gewinn erwächst, unterlasse ich es, hierüber einen Ausweis zu geben, und will nur nebenbei anführen, dass ungefähr in der Hälfte der positiven Fälle die Kinder ausgetragen waren, und dass die andere Hälfte von in verschiedenem Grade frühreifen gebildet wurde.

Es ist schon angeführt worden, dass, um der Frage, ob man die harnsauren Salze in den Nieren der Neugebornen als eine physiologische oder pathologische Ausscheidung zu betrachten habe, näher zu rücken, Fälle constatirt werden müssten, wo vorher ganz gesunde Kinder, die durch ein gewaltsames Ereigniss zu Grunde gegangen, die Nierenkanälchen mit denselben erfüllt zeigten. Unter den 39 positiven Fällen befinden sich nun allerdings 2 , welche dieser Kategorie anzugehören scheinen, und desshalb gewiss erwähnt $\mathrm{zu}$ werden verdienen.

1. Am 6ten Januar 185410 Uhr Morgens wurde in der Poliklinik der Königl. Entbindungs-Anstalt ein gesundes Mädchen geboren; der Practicant fand an demselben keine Veränderung, als er es gegen Abend wieder hesuchte, bei seiner Visite am folgenden Morgen dagegen war es todt, und sollte nach Aussage der Umgebung, um 8 Uhr Abends, also 10 Stunden nach der Geburt, unter nicht auffallenden Erscheinungen gestorben sein; die Verbandstücke des Nabels wurden beträchtlich mit Blut durchtränkt, die Nabelschnur unterbunden gefunden. Die uns übersanidte Leiche war die eines ausgetragenen Kindes, $5 \frac{1}{2}$ Pfund schwer und 18 Zoll lang, und zeigte eine über den ganzen Körper verbreitete wachsgelbe Farbe. Bei der Obduction fand man sämmtliche innere Organe gesund, aber im Zustande der äussersten Blutleere, das Herz von einer geringen Menge flüssigen Blutes erfüllt, đie Lungen ganz blass, die grossen Gefässstämme leer. Die Nieren waren sehr anämisch, beide mit einem nicht unbeträchtlichen Harnsäureinfarcte erfüllt, ihr Gewebe gesund, Harnblase leer, Dickdarm mit Meconium erfüllt. Die Nabelvene und die Nabelarterien enthielten eine geringe Menge flüssigen Blutes.

Wenn man in diesem Falle den offenbar unvermutheten Eintritt des Todes mit dem Ergebnisse der Section vergleicht, so kann man wohl darüber nicht in Zweifel sein, dass es sich hier um eine Verblutung aus dem mangelhaft unterbundenen Nabelstrange gehandelt hat; das in den Organen fehlende Blut konnte nur diesen Weg nach aussen genommen haben, da der Mastdarm noch mit Meconium angefüllt, mithin eine Darmblutung ausgeschlossen war; der Harnsäureinfarct hatte sich also unter Umständen entwickelt, die der Annahme seiner physiologischen Natur günstig erscheinen müssen. 


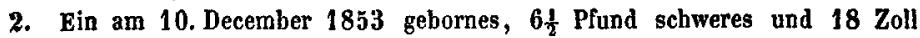
langes Madchen wurde in der Nacht vom 11 ten zum 12 ten; also circa 48 Stunden nach der Geburt, olne vorber Zeichen von Kranksein aufgewiesen zu haben, neben der Mutter, welche es nicht lange vorher an die Brust gelegt hatte und hierüber eingeschlafen war, todt gefunden. Die Section ergab keine den Tod erk]ärende Anomalie, sondern den bei im Bette erstickten Kindern gewölnnlichen Befund, nämlich Ueberfüllung des rechten Herzens und der in dasselbe einmündenden venösen Gefässe mit Blut, ebenso Stauning des Blutes in den lufthältigen Lungen und den Unterleilsorganen; dabei fand sich in beiden Nièren eine nicbt besonders starke, aber deutlich wahrnehmbare Ablagerung von harnsaurem Natron.

Auch diese Beobachtung scheint geeignet, der Ansicht, dass der Harnsäureinfarct zu einer gewissen Zeit bei allen Kindern vorkommt, grösseren Spielraum zu verschaffen, wenigstens erfüllt sie alle Bedingungen, die man an solchen Fall stellen kann, und man wird nicht oft ein noch plötzlicher gestorbenes Kind zur Untersuchung bekommen. Wenn es dennoch fïr mich immer den Anschein gehabt hat, als ob es noch anderer Beweismittel bedürfe, um das Normale des Infaretes zur Evidenz zu bringen, so liegt der Grund davon darin, dass sich auch gegen die angefübrten Fälle gewisse Bedenken meiner Ansicht nach nicht zurückweisen lassen; für beide Kinder bleibt die Möglichkeit, dass irgendwelche abnorme Bedingungen bei anscheinender Integrität des Organismus, so weit diese sich durch Zeichen im Leben und die Section nachweisen lässt, auf das Zustandekommen der Ausscheidung eingewirkt haben; insbesondere verliert der erste Fall durch die Betrachtung an seiner Beweiskraft, dass möglicherweise während der Blutung, die, wegen der Beschaffenheit der Umbilicalarterien und der durch die Respiration bewirkten veränderten Lage der Baucheingeweide, nicht profus gewesen sein, daher nur allmälig zum Tode geführt haben konnte, die Bildung des Infarctes zu Stande gekommen ist.

Die weiteren Beweismittel zur Entscheidung der vorliegenden Frage aber können nur auf zwei Wegen beschafft werden, einmal durch vergleichende Untersuchungen der Nieren jüngerer Thiere, und dann durch genauere Forschungen uber den Urin der Neugebornen. Was den ersten Punkt betrifft, so hat Hodann diesen Weg der Untersuchung betreten, und die Nieren von Hunden, 
Katzen und Kaninchen geprüft, aber kein positives Resultat gewonnen, dagegen fand er bei drei Ferkeln von einem Wurfe im Alter von 14-21 Tagen, welche von einem Hunde todtgebissen worden, in den Nieren selbst und im Nierenbecken ein rosarothes Pulver, welches sehr reich an Harnsäure war; bei einigen 20 todt aus dem Leibe der getödteten Mutter genommenen Ferkeln wiederum Nichts. Ich selbst habe mich auch nur auf die 3 obengenannten Species beschränken müssen, da die Anschaffung junger Thiere, deren Alter nicht aussęrhalb gewisser Grenzen liegen darf, an und für sich Schwierigkeiten genug macht, so dass man nicht darauf rechnen kann, seltenere Arten aufzutreiben. Zuerst verschaffte ich mir ein trächtiges Kaninchen, wartete den Wurf ab, und tödtete vom dritten Tage an jeden Morgen ein Junges, um so den etwaigen Eintritt eines Niederschlages constatiren zu können. A priori war mir nicht wahrscheinlich, dass man hier, wie überhaupt bei Pflanzenfressern, wo der Harn immer eine so entschieden alkalische Beschaffenheit hat, Etwas dem Harnsäureinfarct der Neugebornen Analoges finden würde, doch wäre ja denkbar, dass bei den jungen Thieren, so lange ihre Nahrung nur in Milch besteht, der Urin eine von dem in späteren Zeiten abgesonderten abwejchende Zusammensetzung zeigte. Die Untersuchung hat aber die erste Voraussetzung vollkommen bestätigt; der Urin junger Kaninchen ist schon in den ersten Tagen nach der Geburt trübe und zeigt entschieden alkalische Peaction, in den Nieren kann demgemäss auch keine Ausscheidung von harnsauren Salzen zu Stande kommen.

Auch an den Nieren junger Kälber, über deren Urin ich Nichts weiss, habe ich keine Veränderung wahrgenommen, die mit der gesuchten Erscheinung verglichen werden könnte.

Später untersuchte ich einen Wurf junger Katzen auf dieselbe Weise, wie die Kaninchen, indem ich vom 3ten Tage nach der Geburt an jeden Morgen eine tödtete. Bei diesen ist der Urin klar, blassgelb und reagirt schwach sauer, niemals fand sich auch nur eine Spur von Krystallen in den Nierenkanälchen.

Der Versuch, in dieser Richtung eine Frage an die Natur zu stellen, könnte nur dann zu brauchbaren Resultaten führen, wenn 
es gelänge, umfassende über die verschiedensten Thiere sich ausbreitende Beobachtungen zu sammeln; auf den negativen Befund, der sich hier ergeben hat, kann zunächst wenig Werth gelegt werden, vielmehr könnte er höchstens, wenn sich andere gegen die physiologische Natur des Infarctes sprechende Thatsachen ermitteln liessen, als unterstiutzendes Argument zur Geltung gebracht werden.

Viel näher muss man meines Erachtens der Sache auf dem zweiten angegebenen Wege, nämlich durch Untersuchung des Nierensecretes gesunder neugeborner Kinder kommen, denn ermittelt man die chemische Zusammensetzung des Urins innerhalb der Zeit, wo die Nieren am häufigstèn von harnsauren Salzen erfüllt gefunden worden sind, so muss sich ergeben, ob diese im gesunden Zustande derartig ist, dass die Ausscheidung der Urate in den Harnkanälchen mit ihr in Einklang gebracht werden kann oder nicht; auch haben diejenigen, welche sich mit dem Gegenstand beschäftigt, es an Andeutungen nicht fehlen lassen, welche zeigen, dass sie die Anstellung von Harnanalysen bei Neugebornen als etwas sehr Wünschenswerthes betrachten. Auf den ersten Blick erscheint es nun sehr auffallend, dass die Literatur in Bezug auf derartige Untersuchungen eine so geringe Ausbeute liefert, denn wenn wir von den Angaben von Rayer und Guibourt über den Harn der Säuglinge, welche von Virchow in seiner berühmten Arbeit über Harnsäureabscheidung beim Fötus und Neugebornen *), mitgetheilt sind, abstrahiren, weil sie nicht aus genauen Analysen geschöpft sind, so besitzen wir detailirte Bestimmungen der Harnbestandtheile nur von Prout, Virchow und Picard. Die Analysen der ersten beiden Autoren, welche sich in dem schon citirten Aufsatze des Letzteren S. 188 u. 189. befinden, betreffen beide den Urin todtgeborner Früchte, und Picard (de la présence de l'urée dans le sang. Thèse. Strassbourg 1\$56.) hat sich, wie aus der auf Seite 37 seiner Schrift befindlichen Tabelle hervorgeht, auf die Bestimmung des Harnstoffs im Urin Neugeborner beschränkt. Eine nähere Betrachtung des Gegenstandes lehrt aber sehr bald, dass offenbar die Ausführung eines Vorhabens, wie der Anstellung solcher Harnuntersuchungen, das doch gewiss bei Vielen vorausgesetzt

•) Verhandlungen der Gesellschaft für Geburtsbülfe in Berlin. Jahrgang 2: S. 197. 
werden muss, weil sich schwerlich gerade dieser Zweig der Forschung der allgemeinen Aufmerksamkeit entzogen hat, an den Schwierigkeiten, welche die Beschaffung des dazu nöthigen Materials bietet, gescheitert ist, denn Jahre lang fortgesetzte Versuche, den Urin neugeborner Kinder in für chemische $\mathrm{Zwecke}$ genügender Quantität aufzufangen, haben mich zu der Ueberzeugung gebracht, dass dies ein höchst mühevolles und schwieriges Geschäft ist, welches nur unter besonders günstigen äusseren Umständen zu Resultaten führt, und wenn man vielleicht glaubt, dass man hier, wie beim Erwachsenen, mit leichter Mühe 24stündige Quantitäten erobern kann, so ist man in einem grossen Irrthum befangen. Es ist desshalb nicht zu verwundern, dass man speciell für den vor-

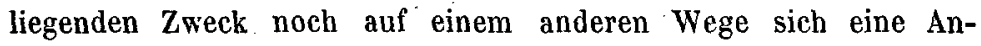
schauung von der Qualität des Nierensecrets zu verschaffen versucht hat, nämlich durch systematische Controlirung der bei den Kindern zur Verwendung kommenden Windeln, in denen sich, wie bekannt, die gelben körnigen Massen des Harnsäureinfarets nach ihrer Ausstossung aus den Nieren leicht wiederfinden lassen; in dieser ${ }_{i}$ Beziehung verdienen die Bemühungen von Hodann, der mit Hülfe eines Seite 22 seiner Schrift auseinandergesetzten Verfahrens sich durch längere Zeit hindurch bei vielen Kindern aus den getrockneten Windeln eine Uebersicht über die Veränderungen des Urins zu verschaffen gewusst hat, die grösste Anerkennung. Die Resultate, die er hierdurch erhielt, waren, dass unter 33 Kindern der gefärbte, mit harnsauren Salzen geschwängerte Urinabgang bei einem Kinde zwischen dem 1sten und 2 ten Tage, bei 5 Kindern zwischen dem 2ten und 8ten, bei 4 zwischen dem 8ten und 14ten, bei je einem zwischen dem 14ten und 21sten und 21sten bis 30sten begann; nachträglicb constatirte er noch den Eintritt béi je einem am 33sten, 35sten und 40sten Tage; die Färbung war durchschnittlich anfänglich scbwach, später intensiver und verlor sich im Zeitraum von etwa 5 bis 6 Tagen. Hiernach waren es von den 33 Kindern 15, also nicht ganz die Hälfte, welche harnsaure Salze durch den Urin ausgeschieden hatten, und es scheint mir ziemlich bedenklich, aus diesem Resultate einen Rückschluss auf die physiologische Natur der Erscheinung machen zu wollen, wie es 
Hodann gethan hat; denn mit Recht fragt man, warum ein gesetzmässiger, normaler Vorgang nicht bei allen Kindern beobachtet werden konnte, und kann unmöglich durch die Antwort, die Hodann darauf ertheilt, zufrieden gestellt werden, dass nämlich 11 kinder schon am 8ten Tage der Beobachtung entrückt wurden, und dass die Färbung bei den anderen erst nach dem 30sten Tage eintrat, mit welchem die Beobachtungen geschlossen wurden, zu welcher Erklärung er noch die Vermuthung hinzufügt, dass vielleicht der Infarct unter noch nicht ermittelten Umständen manchmal dem blossen Auge unmerklich abgehe. Da nämlich in der Mehrzahl der 15 positiven Fälle die Erscheinung zwischen dem 2ten bis 14ten Tage vorkam, so wäre die Annahme gewiss sehr gewagt, dass gerade in 11 Fällen wahrscheinlich die Ausscheidung erst nach dem 8ten Tage, mit dem die Beobachtung aufhörte, aufgetreten sein mag, wie es ebenso künstlich ist, für die übrigen 4 Fălle ein mögliches Auftreten nach dem 30sten Tage zu vermuthen, und es bleibt immer unerklärt, warum nicht einmal 50 pCt. der Kinder bei so sorgfältiger Untersuchungsmethode ein positives Resultat lieferten. Ueberdies erfährt man über den Gesundheitszustand der Kinder nur so viel, dass viele als ganz gesund, einige als krănklich und zwei als sehr krank bezeichnet werden, und gerade diese Angaben sind nicht geeignet, den Beweis für die Normalität des Infarctes zu stützen, denn wenn noch von den $50 \mathrm{pCt}$. ein nicht näher zu bestimmender Abzug, die kränklichen und kranken Kinder umfassend, gemacht werden muss, so resultirt ein Bruchtheil von positiven Fällen, der nicht als Ausdruck eines typischen Vorkommnisses zur Geltung gebracht werden kann.

Meine eigenen Untersuchungen in dieser Richtung sind ohne besonderen Werth, weil von einer Genauigkeit und Sorgfalt, wie sie bei Hodann zu rühmen ist, in der Ausführung derselben nicht die Rede war. Ich habe mich lediglich darauf beschränkt, zeitweise durch Wochen hindurch die Windeln der Neugebornen einer genauen Ocularinspection zu unterwerfen; dabei war es mir allerdings auffallend, dass, so oft ich auf eine mit Harnsäureinfarct geränderte Windel stiess, ich dieselbe einem Kinde angehörig fand, das leichtere oder heftigere Störungen in seinem Befinden zeigte, 


\section{7}

und durch abnorme Beschaffenheit des Nabels, stärkere icterische Hautfärbung, grüngefärbte, übelriechende Ausleerungen u. s. w. Gegenstand speciellerer Aufmerksamkeit geworden war; indessen kann ich, wie gesagt, darauf kein grosses Gewicht legen, weil die Untersuchung nicht systematisch genug betrieben worden ist.

Was nun endlich die Untersuchungen des Urins neugeborner Kinder selbst betrifft, so wiederhole ich, dass ich kein Mittel unversucht gelassen habe, mir dieses Secret in genügender Quantität und brauchbarer Qualität zu verschaffen. Indessen lehrten mich fortgesetzte Bemühungen, dass keines der Verfahren, welche man a priori als hierbei zum Ziele führend betrachten könnte, mit Nutzen eingeschlagen werden kann. Ganz unnütz und nicht ohne erbebliche Bedenken ist zunächst der Katheterismus; denn wenn man wirklich den richtigen Zeitpunkt triff, wo die Harnblase gefüllt ist, was von sehr vielen Zufälligkeiten abbängt, so bewirkt in der Mehrzahl der Fälle schon der Reiz, der bei dem erstẹn Versuche, das Instrument in die Harnröhre einzuschieben, ausgeúbt wird, Contractionen der Blasenmuskeln und Abfluss des Urins, den man dann nicht mehr Zeit hat aufzufangen, oder, wenn man wirklich bis in jene gedrungen ist, so sieht man ganz gewöhnlich den Urin neben dem Katheter und nicht durch denselben abfliessen; da es auf der anderen Seite auf die Dauer nicht gleichgültig sein kann, die Harnröhre eines neugebornen Kindes täglich wiederholt zu reizen, so muss diese Methode als ganz ungeeignet bezeichnet werden.

Ebenso fruchtlos blieben in meiner Hand die Bemühungen, eine für neugeborne Kinder geeignete Form von Harnrecipienten zu construiren; eine ganze Sammlung-von derartigen Apparaten, zum Theil ganz sinnreich von gescheuten Bandagisten erfunden, ist noch in meinem Besitz, obne dass irgend Etwas dadurch erreicht worden wäre, und ich kann die Versicherung geben, dass, mag man einen solchen am Penis allein anbringen, was an und für sich schon sehr schwierig ist, mag man ihn mit einer Oeffnung versehen, in welche Penis und Scrotum hineinpassen, mag man ihn dann mit Schenkelriemen an einem Bauchgürtel befestigen, mag man endlich ein Material nehmen, welches man will, die Re- 
sultate immer dieselben unbefriedigenden sind: entweder nämlich fliesst der Urin nebenbei ab, weil man den Apparat nicht allzufest anschliessend machen kann, wenn man nicht Gefahr laufen will, durch Druck eine ödematöse Infiltration der Genitalien zu erzeugen, oder wenn man Urin im Recipienten findet, so ist er mit Darmausleerungen untermischt, weil man den After nicht so hat ausschliessen können, dass nicht doch Koth mit in die Oeffnung einträte; schliesslich aber wird eine solche Vorrichtung sehr bald durch die fortwährende Verunreinigung von aussen unbrauchbar, das Abnehmen und Befestigen derselben macht so viele in die Ordnung eines neugebornen Kindes störend eingreifende Weitläufigkeiten, dass man auch diesen Weg, sich Urin zu verschaffen, verlassen muss.

Nach vielem Hin- und Herprobiren hat sich mir die Methode als die einzig zweckmässige ergeben, dass man das neugeborne Kind in gewissen Zeitintervallen über einem reinen Nachtgeschirr abhält, ganz so, wie man es mit grösseren Kindern zu machen pflegt. Die günstigste Zeit dazu ist immer bald nachdem das Kind Nahrung zu sich genommen hat, und ich habe mich liberzeugt, dass man sehr bald dasselbe in dieser Beziehung an eine gewisse Ordnung gewöhnen kann, besonders wenn man in der ersten Zeit durch langes Warten und sanfte Reibungen der Blasengegend die Entleerung dieses Organs gewissermaassen erzwingt; bei einiger Aufmerksamkeit passirt es dann nicht allzuhäufig, dass man den richtigen Moment verpasst und der Urin vorher in die Windeln geflossen ist, besonders wenn man die Vorsicht gebraucht, das Kind nur lose einzuwickeln, um ohne Unbequemlichkeit den Versuch, es uriniren zu lassen, öfter wiederholen zu können; natürlich ist auch diese Procedur eine höchst zeitraubende und mühevolle, aber es ist auch darin der Grund zu suchen, dass ich, wie sich weiter unten ergeben wird, nur wenig Material zu Untersuchungen verwerthet habe, aber ich kann diejenigen, welche vielleicht die Absicht haben, diesen Gegenstand weiter zu verfolgen, versichern, dass sie mir als die allein brauchbare erschienen ist.

Ehe ich nun die angestellten Analysen mittheile, will ich zuvor einige Bemerkungen über den Urin todtgeborner Kinder machen. 
Da der Act der Geburt, namentlich bei Unterendlagen der Frucht, häufig Veranlassung giebt, dass die Blasengegend derselben gedrückt wird, so wird der Urin oft inter partum entleert, und man findet bei Obductionen todtgeborner Kinder nur in der Minderzahl der Fälle die Harnblase davon angefüllt; mitunter aber ist sie ganz prall von Urin ausgedehnt. Wenn man inn sorgfältig auffängt, was am besten dadurch geschieht, dass man ihn entweder durch eine in eine kleine künstliche Oeffinung der Blase eingeführte Pifette aufsaugt, oder speciell bei Knaben, dass man die intacte Blase stark zusammendrückt, und ihn so durch die Harnröhre austreten lässt, so hat er immer dieselben Eigenschaften: er ist nämlich sehr hell, nach der Vogel'schen Farbenscala blassgelb, entweder vollständig klar, oder durch Blasenepithelien leicht getrübt, und reagirt stets schwach sauer. Lässt man ihn in einem Gläschen an der Luft stehen, so verdampft die geringe Quantităt, die man überhaupt immer nur erhält, sehr bald vollständig, und es bleibt am Boden des Glases constant ein krystallinischer Rückstand, der, nach der Form der Krystalle zu urtheilen, aus Kochsalz besteht. Schon vor beinahe 3 Jahren, nämlich am 11. Mai 1854, ubersandte ich meinem Freunde, Herrn Dr. Hoppe, damals Arzt am Arbeitshause, einen solchen Urin eines todtgebornen, sonst gesunden Kindes zur Analyse, und derselbe machte mir daruber folgende Mittheilungen: Die ganze Quantität betrug 5,521 Grm. Es fanden sich darin:

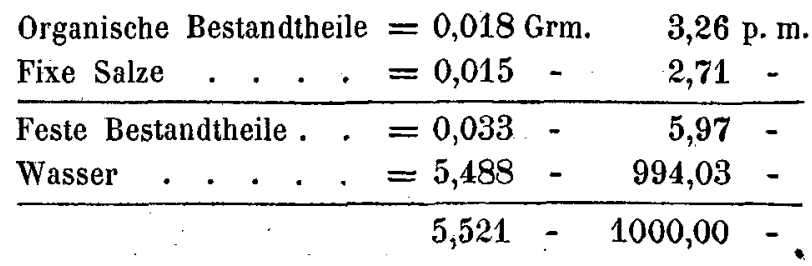

Der gewogene Urin war im Wasserbade getrocknet, dann über Schwefelsäure auf den Recipienten gebracht, der feste Rückstand gewogen, verascht und die rückständige Asche gewogen.

Diese Analyse bestätigt die aus dem Aussehen des Urins todtgeborner Kinder leicht zu entnehmende Vermuthung, dass derselbe arm an festen Bestandtheilen sei, vollkommen, es ist nur zu 


\section{0}

bedauern, dass eine wiederholte Untersuchung eines solchen Harns nicht möglich gewesen ist.

In den Versuchen, die ich $\mathbf{z u}$ derselben Zeit anstellte, Urin aus der ersten Zeit des extrauterinen Lebens zu bekommen, war ich verschiedener Umstände wegen wenig glücklich; ich besitze demzufolge von damals her nur eine, aber sehr genaue Analyse des Harns eines 8 Tage alten gesunden Kindes, welche ich gleichfalls der Güte des Herm Dr. Hoppe verdanke; derselbe erhielt ihn am 19. Mai 1854, und theilte mir die folgenden Resultate darüber mit, die ich hier wörtlich folgen lasse:

Der Urin reagirte sauer, war von Pflasterepithel etwas getrübt und von hellstrohgelber Farbe. Die Menge betrug $112,5 \mathrm{Ccm}$. und zeigte ein specifisches Gewicht von 1,00233. Der Harn wog also $112,362 \mathrm{Grmm}$.

Es wurden 2 gesonderte Portionen untersucht: 19,978 Grm. getrocknet und verkohlt, mit Wasser ausgelaugt, ausgewaschen, die Kohle auf dem Filter getrocknet, gewogen und dann verascht, gaben

$$
\begin{aligned}
& 0,032 \text { Grm. lösliche } \\
& 0,002-\quad \text { unlösliche Salze. }
\end{aligned}
$$

90,711 Grm. im Wasserbade getrocknet, mit kochendem Alkohol von $90 \mathrm{pCt}$. ausgezogen und heiss abfiltrirt. Der ungelöste Rückstand mit Kalilauge gelöst, mit Essigsäure niedergeschlagen, und der geringe Niederschlag mit viel Wasser auf dem gewogenen Filter ausgewaschen, gab 0,010 Grm. Harnsäure. Der heisse Alkobolextract trübte sich beim Erkalten, der Niederschlag wurde beim Erwärmen wieder gelöst. Das Ganze zur Trockne im Wasserbade verdunstet, mit kaltem Alkohol ausgezogen, liess einen Rückstand, dessen einer Theil alle Reactionen des Allantoin gab, nur war der Niederschlag stets amorph. Der kalte Alkoholextract zur Trockne verdunstet, unter der Luftpumpe längere Zeit getrocknet, gab einen äusserst hygroskopischen, zum grossen Theil krystallinischen Rückstand, dieser im Eisbade mit Salpetersäure übergossen, filtrirt und mit saurem Wasser auf dem gewogenen Filter gewaschen und bei $100^{\circ}$ getrocknet, gab $0,076 \mathrm{Grm}$. salpetersauren Harnstoff. 


\section{1}

In Ganzen fanden sich also darin:

\begin{tabular}{|c|c|c|c|}
\hline Harnstoff . . . = & $=$ & \multicolumn{2}{|c|}{ 0,41 p. M. } \\
\hline Harnsäure . . . & $=$ & 0,11 & - \\
\hline Andere organ. Stoffe & $=$ & 6,14 & - \\
\hline Salno / lösliche. & $=$ & 1,60 & - \\
\hline${ }^{\text {Sare }}\{$ unlösliche . & $=$ & 0,01 & - \\
\hline Feste Bestandtheile & $=$ & 8,27 & - \\
\hline Wasser & $=$ & 991,73 & - \\
\hline Harn . . . & & 1000,00 & - \\
\hline
\end{tabular}

Als ich im Sommer 1856 den Gegenstand wieder aufnahm, war mein Augenmerk besonders darauf gerichtet, mir von einem und demselben Kinde durch längere Zeit hindurch Harn zu verschaffen, um seine etwaigen Veränderungen constatiren zu können. Ich wählte desshalb einen kräftigen Knaben, der sich in normaler Weise an der Mutterbrust ernährte, aus, und behielt ihn bis zum 25sten Tage nach der Geburt in meiner Beobachtung. Da mir Zeit und Umstände nicht erlaubten, die Untersuchung des Urins selbst zu machen, so setzte ich mich mit Herrn Prof. Julius Vogel in Halle in Verbindung, der mit der grössten Bereitwilligkeit darauf einging, dieselbe vorzunehmen.

Die Erfahrung, die ich aus meinen früheren Versuchen schon geschöpft hatte, bestätigte sich auch diesmal, dass nämlich bis zum Ablauf des dritten Tages nach der Geburt die Urinsecretion beim neugebornen Kinde eine unerhebliche ist. Nach dieser Zeit fliesst er und floss bei dem Versuchskinde reichlicher, und schienen mir, wenn man eine ganz ungefähre Schätzung der 24stündigen Quantität gelten lassen will, in den nächsten Tagen etwa 3 Unzen täglich secernirt zu werden. Dabei ist es gewiss bemerkenswerth, dass derselbe bis zum 25sten Tage hin in seinem Aeusseren nicht die geringste Veränderung zeigte, er behielt dieselbe blassgelbe Farbe, die er schon beim todtgebornen Kinde aufweist, stets bei, reagirte schwach sauer und setzte beim Erkalten niemals eine Spur von Sediment ab. Bei dem Sammeln der ersten Quantität, welche in einem fest verschlossenen Glase geschah, rechnete ich darauf, dass ich in nicht allzulanger Zeit so viel erhalten würde, um ihn fortschicken zu können, ehe eine erhebliche 
Zersetzung eintrăte; durch Zusatz von Aether, die eine solcbe wohl am besten verhindert, glaubte ich der Analyse selbst zu schaden. Indessen war dieselbe doch schon erfolgt, und Herr Dr. Heidenhain, der ihn im Auftrage des Herrn Prof. Vogel untersuchte, berichtete darüber, dass derselbe neutral reagirte, trübe aussah und bereits faulig roch. Ich füge dennoch hinzu, was die Analyse ergeben hat. Die Harnmenge, die aus der Zeit vom 3ten bis 8ten Tage datirte, betrug $375 \mathrm{Ccm}$., hatte ein sehr niedriges specifisches $\mathrm{Ge}$ wicht, nämlich 1001 und waren in $1000 \mathrm{Ccm}$. 4,5 Grm. Harnstoff, $1,5 \mathrm{Grm}$. Chlornatrium, 0,15 Grm. Schwefelsäure und $0,14 \mathrm{Grm}$. Phosphorsäure enthalten.

Auf den Rath des Herrn Prof. Vogel verfuhr ich beim weiteren Sammeln, um die Zersetzung zu vermeiden, dann so, dass ich die gewonnene Quantität in einem graduirten Glase maass, und dann sofort im Wasserbade zur Syrupsdicke verdampfte: Auf diese Weise erbielt ich bis zum 17ten Tage einen Rüekstand, der $770 \mathrm{Ccm}$. Urin entsprach. Die Analyse dieses Ruickstandes hatte nach den brieflichen Mittheilungen des genannten Herrn Professors folgende Resultate gehabt:

\begin{tabular}{|c|c|c|c|c|c|c|}
\hline $\begin{array}{c}\text { Fester Rückstan } \\
\text { und zwar }\end{array}$ & & & $\begin{array}{r}\text { In } 770 \\
. \quad 4,90\end{array}$ & $\begin{array}{l}\text { Cem. } \\
\text { Grm. }\end{array}$ & $\begin{array}{r}\text { in } 100 \\
6,36\end{array}$ & $\begin{array}{l}\text { Cem. } \\
\text { Grm. }\end{array}$ \\
\hline Harnstoff . . & . & . & . 2,19 & - & 2,84 & - \\
\hline Kochsalz. . & . & . & . 0,69 & - & 0,89 & - \\
\hline Schwefelsäure & . & . & . 0,24 & - & 0,31 & - \\
\hline Phosphorsäure & . & . & . 0,05 & - & 0,06 & - \\
\hline Harnsäure & & . & . 0,24 & - & 0,31 & - \\
\hline
\end{tabular}

„,Den Rest bildeten nicht näher bestimmte Kalk-, Kali-, NatronSalze, Extractivstofie u.s. w. Die Menge der Kalksalze war sehr gering, doch waren neben phosphorsaurem Kalk deutliche Spuren von oxalsaurem Kalk vorhanden. Die gewogene Harnsäure war sehr unrein, mit Haaren, Epithelien, viel Farbstoff und etwas oxalsaurem Kalk gemischt, die geringe Menge des Niederschlages erlaubte jedoch keine genaue Bestimmung derselben in reinem $\mathrm{Zn}$ stande. Die ganze Analyse ergab eine auffallend geringe Menge 
fester Bestandtheile und lässt auf einen sehr geringen Stoffwechsel bei Neugebornen schliessen."

Die letzte Portion, welche ich vom 17ten bis 25sten Tage sammelte, betrug $285 \mathrm{Ccm}$. und hatte Herr Dr. Heidenhain die Güte, mir unter dem 31. August 1856 über diese folgende Mittheilung zu machen: "Die im Schälchen übersandte Masse war sichtlich von fremden Bestandtheilen verunreinigt, daher fällt die Bestimmung des festen Rückstandes jedenfalls zu hoch aus, ebenso die der Extractivstoffe u.s. w., die nach Abzug der genauer bestimmten Stoffe übrig bleiben.

Der. Rückstand von $285 \mathrm{Gcm}$. Harn betrug: 1,8404 Grm. Darin waren:

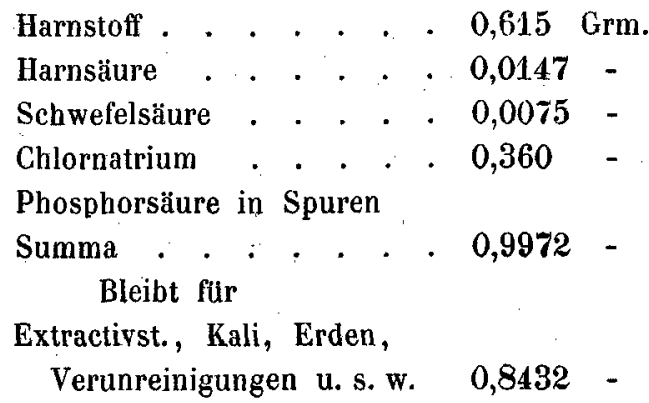

Dass letztere Zahl viel zu hoch ist, versteht sich von selbst. Immerhin bleibt als brauchbares Resultat die sehr geringe Menge aller übrigen genauer bestimmten Stoffe."

Dies sind die Analysen, welche ich den genannten Herren Collegen verdanke. Es liegt auf der Hand, dass dieselben nur als der Anfang ' $z$ zahlreicheren und ausgedehnteren Untersuchungen betrachtet werden können; diese $z u$ veranlassen, war ich nicht im Stande, da ich nicht Zeit genug hatte, fortgesetzt. Urin zu sammeln; auch würde ich die mitgetheilten Resultate noch bis auf Weiteres zurückgehalten haben, wenn ich in nächster Zeit Aussicht bätte, die Sache wieder aufnehmen zu können. Indessen scheint mir das Wenige doch nicht unbrauchbar zu sein; gegenüber den ziemlich verbreiteten Vorstellungen, dass der Urin neugeborner Kinder sehr concentrirt sei, wird durch die Analysen constatirt, dass er auffallend arm an festen Bestandtheilen ist, eine Thatsache, Archiv f, pathol. Anat. Bd, XI, Hft, 3 . 


\section{4}

auf die man schon durch seine blassgelbe Fapbe and sein sehr niedriges specifisches Gewicht geführt wird. Speciell für die Entscheidung der uns beschăftigenden Frage über den Harnsäureinfarct scheint es mir nicht unwichtig, dass in dem benutzten Falle die Armuth des Urins an festen Bestandtheilen sich bis zum 25sten Tage nach der Geburt nicht wesentlich geändert hat; denn bălt man damit zusammen, dass ich an dem Urin niemals ein Sediment, geschweige Etwas, was ausgestossenem Harnsäureinfarct ähnlich gewesen, bemerkt habe, und dass gerade sein Gehalt an Harnsäure so ungemein gering gefunden worden ist, so kann man die Ansicht nicht von der Hand weisen, dass hier eine Bildung von harnsauren Salzen in den Nieren bis zu dem Termin, wo der Urin untersucht worden, nicht gut zu Stande gekommen sein kann, dass aber überhaupt die Beschaffenheit der Flüssigkeit zu der Annahme einer physiologischen Sedimentbildung nicht recht passt. Man könnte freilich einwenden, dass sich hier möglicherweise der Infarct erst nach dem 25sten Tage entwickelt hat, aber gegenüber dieser an sich un wahrscheinlichen Supposition bleibt es jumer auffallend, dass auch die von Herrn Dr. Hoppe gemachte Analyse des Urins von dem 8 Tage alten gesunden Kinde so wenig zu Gunsten einer normalen Sedimentirung in den Nieren spricht; so sehr ich daher auch glaube, dass erst weit zahlreichere Analysen des Urins Neugeborner sichere Anhaltspunkte zur Entscheidung der vorliagenden Frage gewähren können, so kann ich mich, da die Untersuchungen der Windeln mir gleichfalls ein so negatives Resultat ergeben haben, gegenuber den geistreichen Argumentationen Virchow's*), welche in seinen gesammelten Abhandlungen zur wissenschaftlichen Medicin durch die Note 9 zu diesem dort wieder abgedruckten Aufsatze (Seite $861-62$ ) noch eine weitere Ausführung erfahren haben, und selbst im Angesichte der weiter oben von mir mitgetheilten positiven Fülle nach relativ plötzlichem Tode des Kindes nicht von der physiologischen Natur des Harnsăureinfarctes überzeugt halten; vielmehr bin ich der Ansicht, dass der Bildung desselben jedesmal irgendwelche nicht oder wenigstens nicht allein in den veränderten Bedingungen der Existenz begrün*) l. c. 


\title{
295
}

dete Stbrungen im kindlichen Organismus vorhergehen und dass đann auch eine verăndète Beschaffenheit des Urins, besonders ein grơsserer Reíichthum an festen Bestandtheilen desselben mit dem Auftreten jenes coincidiren muss.

\section{XVIII.}

\section{Beitrag zur experimentellen Pathologie der Milz.}

\author{
Von Eduard Jaschkowitz.
}

Die folgenden Zeilen bringen einen kurzen Bericht über einige Experimente, die ich im Laufe des Winters im physiologischen Laboratorium der K. K. Josephs-Akademie zu Wien angestellt habe.

Die anerkannte Bedeutung der Milzkrankheiten war zunächst die Veranlassung meiner Versuche. Es lag mir daran, auf dem Wege des Experiments eine Erkrankung der Milz bervorzurufen, ohne irgendwie den Organismus zu irritiren. 'Seitdem die Wirkungen der Durchschneidungen des sympathischen Nerven am Halse bekannt worden waren, lag der Weg, der hierbei zu betreten war, nicht fern. Bringt die Durchschneidung des Sympathicus am Halse Hyperämie aller von ihm versorgten Partien am Kopfe hervor, so wird, dachte ich, vielleicht dieselbe Erscheinung in Bezug auf die Milz eintreten, wenn man eine Durchschneidung der zu diesem Organe gehenden Aeste des Sympathicus vornähme. Ich beschloss daher, den Plexus lienalis zu durchschneiden und zunächst die Einwirkungen dieser Operation in Bezug auf die Milz zui beobachten, um, wenn diese etwas Positives ergäben, sekundäre Erscheinungen, die in Folge dessen vielleicht im Blute auftreten würden, zu verfolgen.

Von den ausgeübten Operationsmethoden hat sich mir am besten die bewahrt, dass ich vermittelst eines Transversalschnittes 